\title{
Resistance of composite films based on polystyrene and graphene oxide
}

\author{
A. R. Khairullin ${ }^{1}$, M. N. Nikolaeva ${ }^{1}$, A. N. Bugrov ${ }^{1,2}$ \\ ${ }^{1}$ Institute of macromolecular compounds RAS, Bolshoy pr. 31, \\ 199004 Saint Petersburg, Russia \\ ${ }^{2}$ Saint Petersburg Electrotechnical University "LETI", \\ ul. Professora Popova 5, 197376 St. Petersburg, Russia \\ ahairullin@hotmail.com,marianna_n@mail.ru, alexander.n.bugrov@gmail.com
}

\author{
PACS 36.20.-r; 68.65.Pq; 71.20.Rv; 72.80. Tm; 74.78.-w \\ DOI 10.17586/2220-8054-2016-7-6-1055-1058
}

\begin{abstract}
Polystyrene films prepared by radical polymerization can conduct electric current in metal-polymer-metal structures with film thicknesses of up to 20 nanometers. Films of polystyrene and graphene oxide composite with thickness up to 3 micrometers, synthesized in similar conditions have the same electric properties. This effect is explained by presence of highly conductive graphene oxide inclusions in the dielectric polystyrene matrix.
\end{abstract}

Keywords: graphene oxide, polystyrene, composite, conductivity.

Received: 23 August 2016

Revised: 1 September 2016

\section{Introduction}

The interest in the study of conducting polymers with dielectric properties can be explained by the possibility their use as components of electronic devices in various fields [1-4]. It is known [1-8] that thin films of dielectric polymers are able to conduct electricity below the electric field breakdown. Whereas doped polymers have conductivity levels similar to semiconductors. It is shown that films of such materials have metallic type conductivity [8-14]. Considering chemical structure of the polymers, conductivity of such polymer films can be selectively altered. It is necessary to vary the physicochemical properties of polymeric films to determine the factors influencing on conductive properties of dielectric polymers. Different additives were introduced into polymer films during their syntheses. The aim of this work was to create a new polymeric composite material with high conductivity. Polystyrene was used as a polymer matrix because electrization is most pronounced for polymers with high resistivity $[15,16]$. The conductivity in polystyrene films appears in its turn due to the electrization of polymer by a metal substrate. In this instance, graphene oxide (GO) was used as the conducting additive. This material after heat treatment in an aqueous medium [17] becomes a semiconductor, and may even have zero bandgap. After further special treatment as it was done for zirconium oxide in [18], GO may form covalent bonds with polystyrene [19]. It is well known, that a high-conductivity state is observed in such composite films [19]. Also, according to Josephson, the current-voltage characteristics phenomenon of superconductivity was also shown $[20,21]$.

\section{Experimental}

Hummers wet chemical method was used to produce graphene oxide [22]. Graphite was used as an initial material for GO production. After series of chemical reactions in liquid medium, the prepared GO was extracted from an aqueous suspension by aerobic drying at room temperature. Surface modification of GO was carried out using the method described in our previous work [19]. Polystyrene and polymer-inorganic composites were synthesized under identical conditions by free radical polymerization in solution. Styrene in an amount of $0.5 \mathrm{ml}$ and $1 \%$ of 2,2/-azobis(2-methylpropionitrile) (AIBN) by weight of the monomer were dissolved in $0.5 \mathrm{ml}$ of toluene. In the case of the composite, we took $0.47 \mathrm{~g}$ of styrene and $4.9 \mathrm{mg}$ of AIBN as an initiator, and then added them to the dispersion of GO particles in toluene. Then the reaction mixtures with the initial GO content of $1 \mathrm{wt} . \%$ and without filler were placed into ampoules, which were purged with argon for $10 \mathrm{~min}$ and sealed. Polymerization was carried out for 35 hours at $70^{\circ} \mathrm{C}$, every hour for 15 minutes the ampoules were sonicated. Part of the polymerization mixture was precipitated by methanol and dried in vacuo to constant weight. This was done to determine polystyrene yield, which reached $90 \%$ by weight. The molecular weight of the synthesized polystyrene determined by intrinsic viscosity values was 38000 . 
The distribution and agglomeration of GO particles in polystyrene matrix was investigated by Zeiss Supra 55VP field emission scanning electron microscope (Germany).

Films of the polymer composite were deposited on copper electrodes through the dispenser by casting from a solution of $1 \%$ by weight. The thicknesses of the films were controlled by the interference microscope using the method described in [2-4]. Current-voltage characteristics of the metal-composite-metal structures were obtained by the modified two-probe method [2-4]. The area of the lower electrode was $1 \mathrm{~cm}^{2}$, and the upper one $1 \mathrm{~mm}^{2}$. Measurements were carried out at a pressure not more than $1 \mathrm{~kg} / \mathrm{cm}^{2}$. The resistive nature of obtained current-voltage characteristics exclude the presence of a breakdown in films which have been investigated.

\section{Results and discussion}

It was found that the amount of GO particles covalently coupled to polystyrene matrix is about $0.02 \mathrm{wt} . \%$ (4 vol.\%), according to the SEM data of the composite films (Fig. 1). The distance between individual GO particles in polymer matrix ranges from tens to hundreds of microns. The inclusions of GO are distributed rather evenly in the polystyrene matrix due to the formation of chemical bonds between the vinyl groups of modified graphene and polystyrene. It should be noted that the lengths of individual GO particles reach more than 10 micrometers and few hundred nanometers in width. Furthermore, GO particles do not overlap, as can be seen in SEM micrographs (Fig. 1). The particles of GO are arranged parallel to the substrate and do not form a multilayer assemblies, which typical for graphene due to $\pi-\pi$-stacking [23]. This fact explains the little thickness of the composite at which conductivity is observed. As a result, high conductivity can be seen nearby GO particles. Composite films with thicknesses less than $3 \mu \mathrm{m}$ conduct electrical current, at larger thicknesses, the composite material has dielectric behavior, even in areas with GO inclusions. Polystyrene without graphene oxide, synthesized under the same conditions, conducts only with thickness up to $0.02 \mu \mathrm{m}$.

Temperature dependences for the resistance, which were obtained for the composite films at thicknesses up to $0.2 \mu \mathrm{m}$, indicate that the samples have metallic conductivity on the entire surface area (Fig. 2).
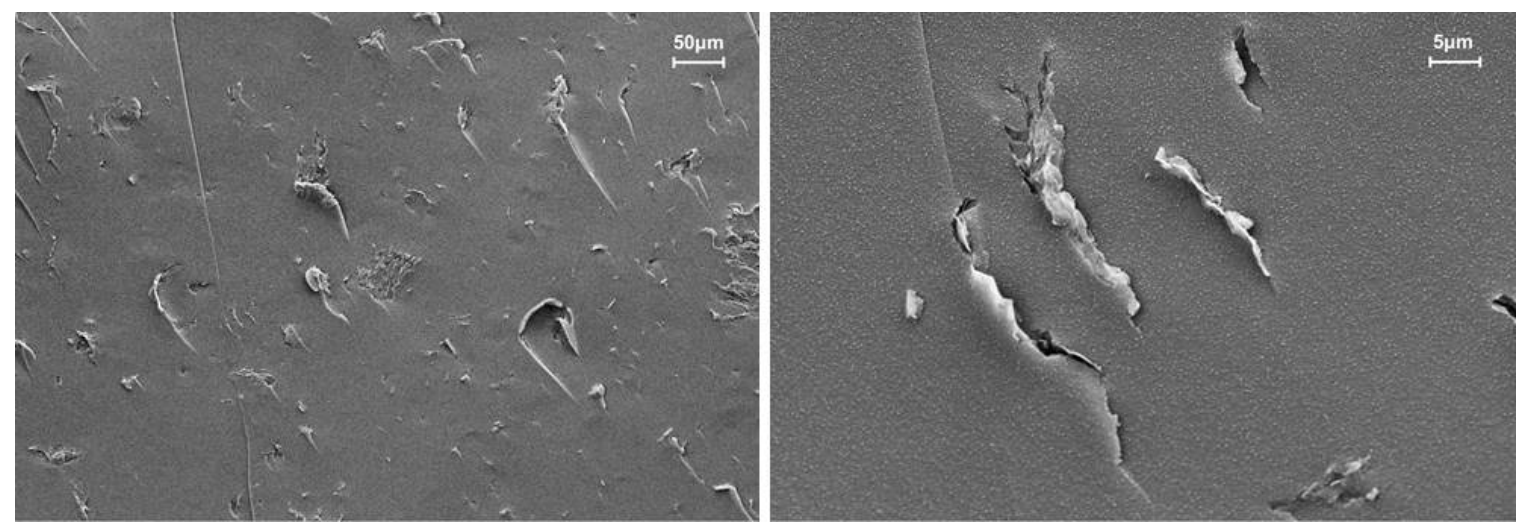

FIG. 1. Micrograph of the composite surface

Films with thickness $0.2 \mu \mathrm{m}$ and above (up to $3 \mu \mathrm{m}$ ) are non-uniform in conductivity values. Areas of the films close to GO particles have low resistivity, but larger part of the surface without inclusions has higher resistance from a few ohms to hundreds of kilo-ohms and higher. So resistance temperature dependences were radically different for various parts of the film. Polystyrene surfaces without GO demonstrated infinite resistance at liquid nitrogen temperatures, while areas filled GO were changed into a highly-conductive state. The resistance decreased by 5-6 orders of magnitude when the temperature was lowered from room temperature to the boiling point of liquid nitrogen (Fig. 3). Such resistance-temperature dependences for film areas with GO particles are anomalous. This effect requires further investigation. It should also be noted that composite films based on polystyrene and GO particles have Josephson current-voltage characteristics over a wide temperature range from liquid helium to room temperature, according to results obtained previously [20,21]. This effect indicates the possible existence of superconducting channels in the composite films. Since in our case in-situ introduced graphene oxide undergoes various deformations, then it seems to be possible manifestation of the previously theoretically predicted effect of superconductivity in graphite [24-27]. 


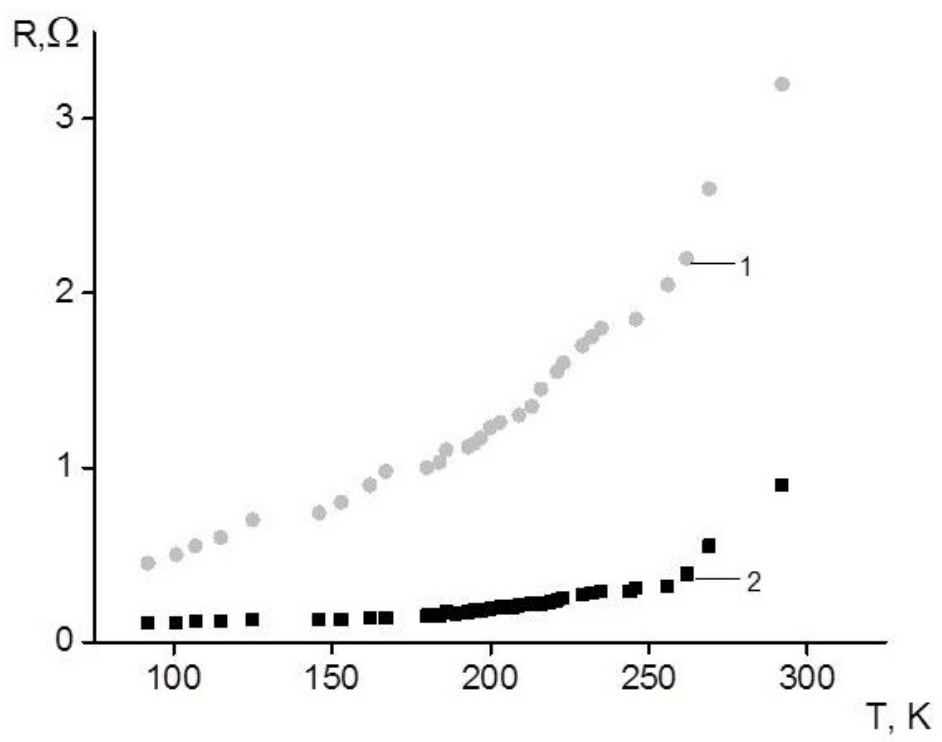

FIG. 2. Temperature dependences of the resistance for the composite films with a thickness of $0.2 \mu \mathrm{m}$ : 1-areas of the polystyrene film without graphene oxide inclusions; 2-areas of the polystyrene film with particles of graphene oxide

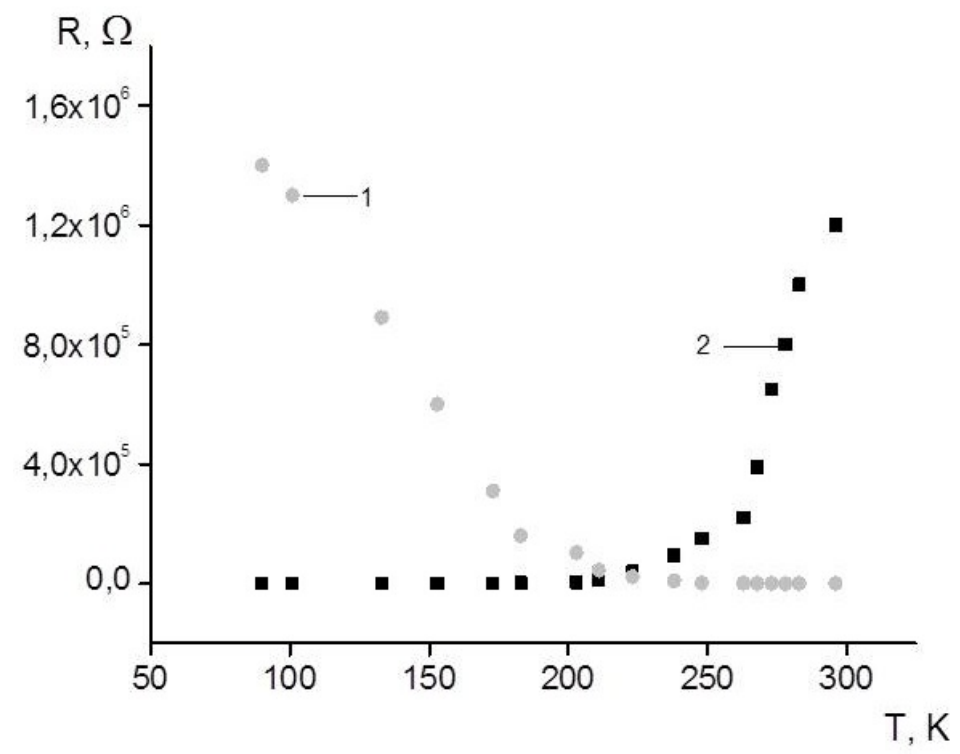

FIG. 3. Temperature dependences of resistance for the composite films: 1 -areas of $0.3 \mu \mathrm{m}$ thick polystyrene films without graphene oxide inclusions; 2-areas of $2 \mu \mathrm{m}$ thick polystyrene films with particles of graphene oxide

\section{Conclusions}

As a result of chemical synthesis, composite films based on polystyrene covalently linked to 0.02 wt.\% of graphene oxide particles were obtained.

The resulting composite films have high conductivity in areas adjacent to graphene oxide inclusions, while the remaining film areas are highly-resistant above $0.3 \mu \mathrm{m}$ thickness. It is important to note that decrease in resistance value near graphene oxide inclusions for the 0.2 to $3 \mu \mathrm{m}$ composite films can vary by several orders of magnitude in the temperatures ranging from the boiling point of liquid nitrogen to room temperature. For $3 \mu \mathrm{m}$ thick films, the resistance values can vary by 6 orders of magnitude. Thinner composite films show resistance temperature dependence similar to that of metallic type conductivity. 


\section{Acknowledgement}

We are grateful to T.D. Ananeva (Institute of Macromolecular Compounds, Russian Academy of Sciences, St. Petersburg) for taking part in the synthesis of composites. We deeply appreciate E. M. Ivankova (Institute of Macromolecular Compounds, Russian Academy of Sciences, St. Petersburg) for scanning electron microscopy measurements.

\section{References}

[1] Ionov A.N., Dunaevskii M.S., et al. The dependence of polymer conductivity on the work function of metallic electrodes. Ann. Phys., Berlin, 2009, 18, P. 959-962.

[2] Nikolaeva M., BoikoY., Martynenkov A. Supramolecular structure and conductive properties of dielectric polymers in metal/polymer/metal systems. Int. J. Polym. Mat., 2013, 62(13), P. 706-710.

[3] Nikolaeva M.N., Anan'eva T.D., et al. Influence of chemical structure and chain length on conducting properties of dielectric polymers in metal/polymer/metal structures. Rus. J. Appl. Chem., 2013, 86(5), P. 756-759.

[4] Nikolaeva M.N., Martynenkov A.A., et al. Resistance of dielectric polymer films with fillers in metal-polymer-metal systems. Rus. J. Appl. Chem, 2014, 87(5), P. 646-650.

[5] Deepak A., Shankar P. Exploring the properties of lead oxide and tungsten oxide based graphene mixed nanocomposite films. Nanosystems: Physics, Chemistry, Mathematics, 2016, 7(3), P. 502-505.

[6] Arhangorodsky V.M., Ionov A.N., et al. Ultra-high conductivity in the oxidized polypropylene at room temperature. JETP Letters, 1990, 51(1), P. 56-62. (in Russian)

[7] Ionov A.N., Nikolaeva M.N., Rentzsch R. Local distribution of high-conductivity regions in polyamidine films. JETP Letters, 2007, 85, P. $636-638$

[8] Ionov A.N., Nikolaeva M.N., Rentzsch R. Metallic conductivity in a polyamidine film. Physica C, 2007, 460-462, Part 1, P. 641-642.

[9] Nikolaeva M.N., Aleksandrova G.P., Martynenkov A.A. Effect of electrization on molecular mobility in gold nanocomposites based on arabinogalactan. Rus. J. Phys.Chem. (A), 2012, 86, P. 812-815.

[10] Nikolaeva M.N., Aleksandrova G.P., Ionov A.N. Correlation between the electrification and molecular mobility of noble metal nanocomposites based on arabinogalactan. Rus. J. Appl. Chem., 2011, 84, P. 450-453.

[11] Ionov A.N., Svetlichnyi V.M., Rentzsch R. Electron transport in metal-polymer-metal systems. Physica B: Cond. Matter, 2005, 359-361, P. 506-510.

[12] Rentzsch R., Ionov A.N., Nikolaeva M.N. Spreading resistance microscopy study of polyamidine thin films. Phys. Stat. Sol. (c), 2006, 3(2), P. 275-279.

[13] Ionov A.N., Nikolaeva M.N., Pozdnyakov O.F., et al. Molecular structure of poly(siloxaneimide) films and the rate of charge relaxation. Polym. Sci. (A), 2008, 50, P. 174-182.

[14] Ionov A.N., Rentzsch R., Nikolaeva M.N. Metallic conductivity in a polyamidine film. Phys. Stat. sol. (c), 2008,5 , P. $730-734$.

[15] Lowell J. and Rose-Innes A.C. Contact electrification. Adv. Phys, 1980, 29(6), P. 947-1023.

[16] Duke C.B., Fabish T.J. Charge-induced relaxation in polymers. Phys. Rev. Lett., 1976, 37(16), P. 1075-1078.

[17] Mikoushkin V.M., Shnitov V.V., Nikonov S.Yu. et al. Controlling Graphite Oxide Bandgap Width by Reduction in Hydrogen. Tech. Phys. Lett., 2011, 37(10), P. 942-945.

[18] Bugrov A.N., Vlasova E.N., Mokeev M.V. et al. Distribution of zirconia nanoparticles in the matrix of poly(4,4/oxydiphenylenepyromellitimide). Polym. Sci. Ser. B., 2012, 54, P. 486-495.

[19] Nikolaeva M.N., Bugrov A.N., et al. Conductive properties of the composite films of graphene oxide based on polystyrene in a metalpolymer-metal structure. Russ. J. Appl. Chem., 2014, 87(8), P. 1151-1155.

[20] Ionov A.N. Josephson current-voltage characteristic of a composite based on polystyrene and graphene oxide. Tech. Phys. Lett., 2015, 41(7), P. 651-653.

[21] Ionov A.N. Josephson-Like Behaviour of the Current-Voltage Characteristics of Multi-graphene Flakes Embedded in Polystyrene. J. Low Temp. Phys., 2016, 182(3/4), P. 107-114.

[22] Hummers W., Offeman R. Preparation of graphitic oxide J. Am. Chem. Soc., 1958, 80(6), P. 1339-1339.

[23] Hazarika M., Jana T. Graphene nanosheets generated from sulfonated polystyrene/ graphene Nanocomposite. Cmpos. Sci. Tech., 2013, 87, P. 94-102.

[24] Heikkilä T., Kopnin N.B., Volovik G. Flat bands in topological media. JETP Lett, 2011, 94, P. $233-237$.

[25] San-Jose P., Prada E. Helical networks in twisted bilayer graphene under interlayer bias. Phys. Rev. B, 2013,88, P. 121408.

[26] Uchoa B., Barlas Y. Superconducting states in pseudo-Landau levels of strained graphene. Phys. Rev. Lett., 2013, 111(1-5), P. 046604.

[27] Bianconi A., Jarlborg T. Lifshitz ons and zero point lattice fluctuations in sulfur hydride showing near room temperature superconductivity. Nov. Supercond. Mater, 2015, 1, P. 37-49. 